\title{
TRAYECTORIAS EDUCACIONALES Y LABORALES DE LOS TÉCNICOS EN CHILE
}

\section{Motivación}

La formación de técnicos constituye un dilema dentro del discurso acerca de educación superior en Chile. Existe una disyuntiva entre la necesidad de técnicos que contribuyan al aumento de la productividad -dado los cambios en la economía, producto del crecimiento sostenido de la última década-y su valoración en términos del salario esperado al egreso.

En Chile persiste una estructura atípica en la formación de capital humano si comparamos con países más desarrollados. Existen seis estudiantes en carreras universitarias por cada uno en carreras técnicas de nivel superior. Esto se puede explicar por la ausencia histórica de una política de financiamiento público hacia el sector y la fuerte brecha de remuneraciones entre profesionales universitarios y técnicos de nivel superior, hechos que llevan a la percepción de que la formación técnica es el comúnmente denominado "pariente pobre de la educación superior". Pese a esta impresión, de acuerdo con información de Futuro Laboral ${ }^{3}$, un estudiante con cinco años de estudio en una universidad de baja calidad bien podría ganar menos que su contraparte que ha estudiado en la misma área por dos años en un centro de formación técnica (CFT) de estándar aceptable.

En Chile esta discusión es un fenómeno incipiente. En los últimos años, numerosos estudios han abarcado el tema, orientados a diferentes ámbitos y referidos principalmente al desempeño de los CFT a la luz de la acreditación y de una mirada más de mercado, analizando la competencia y la estructura del sistema.

Estas investigaciones e informes han hecho posible complementar el análisis del sector -que se basa en las cifras del

3 www.futurolaboral.cl 
sistema como un todo- con los datos que surgen de la observación de las instituciones que componen el sistema. Sin embargo, la falta de información y mediciones relevantes ha restringido en gran parte abordar la problemática de manera más integral.

Este trabajo, en cambio, pretende dar una mirada distinta a los diagnósticos realizados hasta ahora, analizando -a la luz de los acontecimientos que han enmarcado a la educación técnica- si constituye verdaderamente una opción válida de estudio, visto el proceso desde la perspectiva del número de alumnos que deciden por ella. Construye las trayectorias educacionales y laborales de los que optaron por ésta e intenta explorar también si estas trayectorias han reproducido o reproducen inequidades. Es decir, si el sistema-como está estructurado- contribuye a la segmentación y estratificación social, y-lo más importante- de qué manera.

Iniciamos este estudio trazando las trayectorias educacionales del individuo a fines de la educación media. Tomamos en cuenta este punto de partida porque es normalmente el momento en que la persona toma decisiones acerca de su educación superior. No indagamos sobre los factores que afectan la decisión del individuo de seguir estudiando, ya que es tema de otra investigación y excede los alcances de este trabajo. Queremos explorar si en este recorrido que escogió la persona hubo patrones de desigualdades educacionales que determinaran de alguna manera la senda escogida. Intentamos una respuesta a partir de la relación entre el origen social del individuo y su educación. Para esto, replicamos un modelo de transición, con datos extraídos de la encuesta Panel Casen 1996-2001-2006, con la que podemos armar las transiciones educativas de los individuos y estudiar en qué medida sus factores de origen social influyeron en su trayectoria. También construimos transiciones laborales, lo que permite obtener un enfoque dinámico de constitución de procesos

4 Encuesta longitudinal que analizó una muestra de hogares de las regiones III, VII, VIII y Metropolitana. Permitió realizar un seguimiento de familias en 1996, 2001 y 2006, así como los factores que los llevaron a entrar, salir o permanecer en la pobreza. Mientras la encuesta Casen da como resultado una "fotografía" de un determinado momento, la Panel Casen 1996, 2001, 2006 muestra la "película" a lo largo de 10 años. 
más que de caracterización de determinados grupos en un punto del tiempo.

\section{Análisis de los datos}

La encuesta Panel Casen es representativa de los hogares privados de cuatro regiones (III, VII, VIII y Metropolitana), los que a su vez representan aproximadamente $60 \%$ de la población del país.

La base de datos contiene información sobre los miembros originales de la muestra y de personas que fueron encuestadas en las olas posteriores a la primera, porque vivían en un hogar con algún miembro de la muestra original de 1996, siendo en total 20.948 personas. Para el propósito de este estudio sólo se siguió a las personas originales de la muestra que fueron encuestadas en las olas anteriores. Esto es, personas encuestadas en las olas 1996, 2001 y 2006. La muestra longitudinal consta de 10.287 individuos, ponderada por el factor de expansión asociado a las personas que formaron parte de la muestra a lo largo de todo el periodo de estudio.

Antes de comenzar con el modelo es importante aclarar la metodología empleada para la obtención de las trayectorias. Es interesante saber si, en un año específico, una persona ingresó a estudiar en un determinado nivel o se graduó en el mismo. Lamentablemente, debido a las limitaciones de la manera en que se dispone la información, las trayectorias se van armando cruzando variables de asistencia y variables de escolaridad, para crear la secuencia de historia educativa del individuo. Los datos presentan información sólo de niveles o cursos (nivel actual o último cursado, dependiendo si asiste o no a un establecimiento educativo) en los tres años de la toma de la encuesta Casen, 1996, 2001 y 2006. Para los años intermedios que contiene el panel de datos (1997 a 2000, para la muestra de 1996-2001; 2002 a 2005, para la muestra de 20012006) no se cuenta con la información sobre los niveles educativos, sino sólo sobre la asistencia en esos años en cuestión y de las razones de inasistencia, en el caso de no estar ese año en el sistema. Por lo tanto, para construir la historia educacional de los individuos de manera consistente se cruzaron, en primer lugar, las variables de 
tipo de educación ${ }^{5}$ de los años 1996, 2001 y 2006, lo que nos da una idea inicial de la historia educativa del individuo. En segundo lugar, y dado este cruce preliminar, se analizaron los años intermedios a los mencionados, si el individuo asistía o no al establecimiento educacional y las razones por las cuales no asistía, si este fue el caso. Con esta información se comienzan a armar las trayectorias. Por último, para tener consistencia en la historia educativa, la última variable de control se vincula con los años de escolaridad, la cual define el nivel educativo que alcanzó el individuo hasta la fecha de medición (también 1996, 2001 y 2006).

La trayectoria lograda para los individuos de 16 a 18 años que asistían en 1996 a cuarto medio de la modalidad científico humanista (EMCH) y técnico profesional (EMTP) se muestra en el gráfico 1. La primera transición ${ }^{6}$ al año 1997, con el ingreso a educación superior (centros de formación técnica [CFT], institutos profesionales [IP] y universidades [Ues]) o abandonando el sistema. La siguiente transición se realiza en el año $2002^{7}$, en la cual las opciones son completar el ciclo iniciado en la trayectoria anterior, continuar en el sistema (IP o Ues) o abandonarlo. Por último, el punto de cierre es el año de la última medición, 2006, con las mismas opciones de completar ciclo, asistir o abandonar el sistema.

5 Los tipos de educación definidos en la encuesta Panel Casen son: educación preescolar o parvularia, preparatoria (sistema antiguo), educación básica, escuela especial (diferencial), humanidades (sistema antiguo), educación media científico humanística, técnica, comercial, industrial o normalista (sistema antiguo), educación media técnico profesional, centro formación técnica incompleta (sin título), centro formación técnica completa (con título), instituto profesional incompleta (sin título), instituto profesional completa (con título), educación universitaria incompleta (sin título), educación universitaria completa (con título), universitaria de postgrado, educación básica de adultos, educación media de adultos, ns/nr ninguno.

6 Partiendo de 12 años de escolaridad efectiva hasta 1996.

7 Con 16 años de escolaridad alcanzados previamente en 2001. 
Gráfico 1: Trayectorias educacionales cohorte 1996, cuarto medio ambas modalidades (edad 16 a 18)

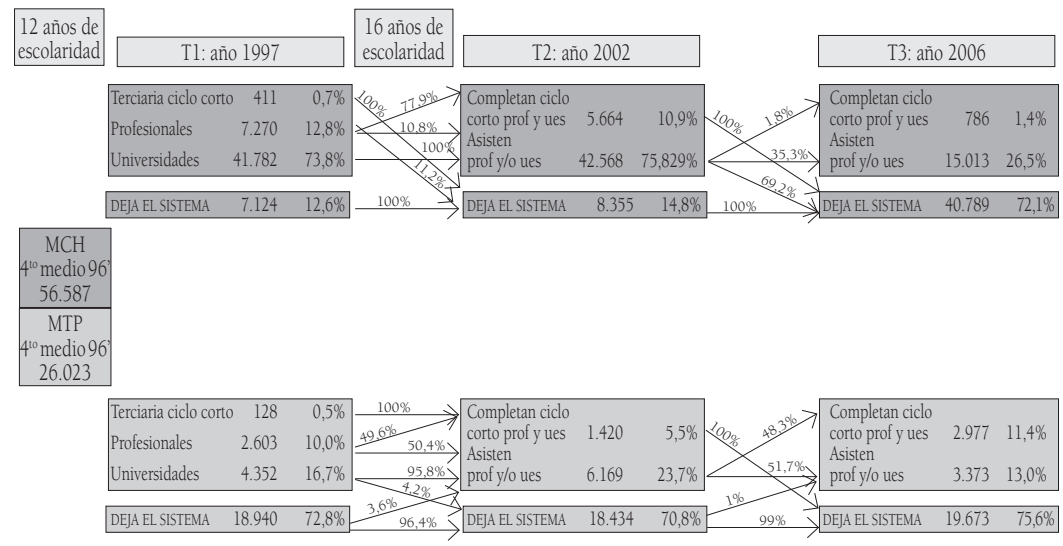

Nos interesa evaluar una dinámica en el tiempo y para eso se requiere de un grupo de comparación. Por lo que, siguiendo la misma metodología de construcción de las trayectorias para la cohorte 1996, se estimó conveniente elaborar una similar para la cohorte 2001, es decir, alumnos que en ese año tenían de 16 a 18 años y asistían a cuarto medio de ambas modalidades, científico humanista y técnico profesional.

Esta comparación permitirá evaluar la consistencia de los estimadores que obtengamos de los modelos.

La trayectoria lograda para los individuos de 16 a 18 años que asistían en 2001 a cuarto medio de la modalidad científico humanista y técnico profesional es la siguiente: 
Gráfico 2: Trayectorias educacionales cohorte 2001, cuarto medio ambas modalidades (edad 16 a 18)

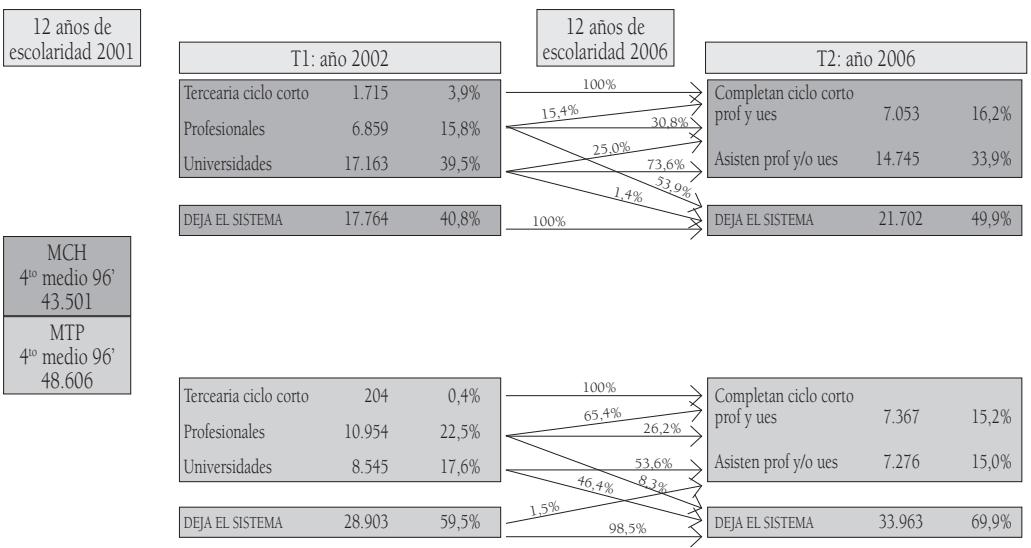

\section{Variables utilizadas}

Antes de presentar el modelo es importante revisar una breve descripción de las principales variables contempladas para armarlo:

Se observa en las trayectorias que vimos anteriormente que, en general, las proporciones de individuos que acceden a educación superior se equiparan en la cohorte de 2001. Esto puede deberse al incremento de la oferta educativa o a mayores posibilidades de financiamiento, entre otras razones, lo que corresponde al incremento observado en el porcentaje de cobertura bruta de educación superior.

Hay que distinguir en ambas cohortes que la decisión de ingresar a universidades como opción de educación superior siempre es la más observada.

Respecto del nivel educacional alcanzado por los individuos de las distintas cohortes, observamos que en 1996 un 7\% igualó el nivel de escolaridad de su padre y un 93\% lo superó. Las cifras son similares si analizamos el nivel de escolaridad de la madre. Para la cohorte 2001 vemos que sólo un 1,3\% tuvo un nivel de escolaridad menor que su padre, el 99,3\% mayor, mientras que el 100\% superó a su madre (los niveles educacionales de las madres en general eran más bajos que los de los padres). 
En vista de la información presentada y la evidencia teórica, las variables que incluirá en el análisis son: escolaridad del padre (epadre) $)^{8}$, ingreso total per cápita en miles de pesos chilenos, corregido por IPC $^{9}$ dependiendo del año en que se analice la trayectoria y una dummy (rm) que indica si el individuo está localizado en la Región Metropolitana o en otras regiones. Dada la disponibilidad de los datos y para evitar la pérdida de información por no respuesta, no se tuvo en cuenta la variable categoría ocupacional de los padres, ya que hubo una gran proporción de no repuesta, lo que restringía la muestra aún más (aunque se modeló y sus resultados fueron estadísticamente no significativos).

\section{Aplicación modelo logit multinomial}

Existe una gran tradición acerca de analizar las carreras educativas como transiciones secuenciales entre grados o niveles de educación. Este tipo de modelo fue popularizado por Mare $(1980,1981)$ en su trabajo pionero, que mostró las ventajas de usarlo, transformándose en el modelo estándar de la investigación empírica de la estratificación social presente en las trayectorias educativas. Como también describen Breen y Jonsson (2000), las ventajas de este modelo en el estudio de este tipo de trayectorias son diversas. Se enfoca en el análisis de los log odds (logaritmo de los ratio odds) ${ }^{10}$ de hacer la transición del nivel $t+1$, condicional a haber alcanzado el nivel t. Estos log odds son usualmente tomados como lineales en un set de $J$ variables exógenas $X_{j}$, cuyos valores varían entre individuos y posiblemente entre transiciones. Como el modelo de Mare está basado en los odds ratio, insensibles a las distribuciones marginales, los parámetros del modelo no están afectados por expansiones agregadas o contracciones en el sistema educacional. De esta manera, el modelo refleja los efectos "puros" de las variables exógenas. Además, estos modelos de transiciones secuenciales son fáciles de estimar. Breen y Jonsson (2000) extienden este argumento suponiendo que los individuos no progresan en sus

8 Construida a partir de las variables tipo de educación y último curso completado.

9 Índice de Precios al Consumidor.

10 El ratio odds es el cociente entre la probabilidad de que un evento suceda y la probabilidad de que no suceda. 
carreras a través de un sistema educativo exclusivamente de una manera secuencial. Dichos autores extienden el modelo de Mare de manera de tomar en cuenta sendas educativas no secuenciales y estudian la inequidad empíricamente, reemplazando el modelo logit binario por un logit multinomial, metodología que se adopta en este trabajo, dado que, efectivamente, las opciones de los individuos al terminar la enseñanza media, como hemos visto en el análisis de las trayectorias, no es producto de un recorrido secuencial, sino que existe un árbol de decisión que no es lineal para el individuo en cada punto de decisión (inicio de la transición).

\section{Resultados principales}

La tabla 1 nos muestra un resumen del efecto de las variables incluidas en el análisis en las distintas trayectorias:

Tabla 1: Ratio odds estimación del modelo. Cohorte egresados $4^{\text {to }}$ Medio Científico Humanista en 1996

\begin{tabular}{|c|c|c|c|c|c|c|c|}
\hline & \multicolumn{3}{|c|}{ Tl 1996} & \multicolumn{2}{|c|}{ T2 2002} & \multicolumn{2}{|c|}{ T3 2006} \\
\hline & $\begin{array}{c}\text { Ingresa a } \\
\text { CFT }\end{array}$ & \begin{tabular}{c|} 
Ingresa \\
a IP
\end{tabular} & Ingresa a Ues & $\begin{array}{c}\text { Completa } \\
\text { ciclo }\end{array}$ & $\begin{array}{l}\text { Continúa } \\
\text { Ues o IP }\end{array}$ & $\begin{array}{c}\text { Completa } \\
\text { ciclo }\end{array}$ & $\begin{array}{l}\text { Continúa } \\
\text { Ues o IP }\end{array}$ \\
\hline \multirow[b]{2}{*}{$\mathrm{rm}$} & $6.4 e+13$ & $155.1408^{*}$ & $* * 24.0600^{* * *}$ & & $4.5707^{* * *}$ & 0.0000 & $0.0279 * * *$ \\
\hline & $(1.0 e+17)$ & $(11.1869$ & ) (1.1555) & & $(0.2030)$ & $(0.0000)$ & $(0.0009)$ \\
\hline \multirow{2}{*}{ epadre } & 0.0000 & $0.7105^{* *}$ & \begin{tabular}{l|l}
$*$ & $0.1261^{* * *}$ \\
\end{tabular} & 0.0000 & $0.7338^{* * *}$ & $4.1 e+27$ & $1.7097^{* * *}$ \\
\hline & $(0.0000)$ & $(0.0159$ & $(0.0059)$ & $(0.0000)$ & $(0.0140)$ & $(1.4 e+34)$ & $(0.0861)$ \\
\hline \multirow{2}{*}{$\begin{array}{l}\text { Ingreso } \\
\text { total per } \\
\text { cápita }\end{array}$} & $1.0506 * * *$ & $1.0723^{* *}$ & \begin{tabular}{l|l}
$*$ & $1.0633^{* * * *}$
\end{tabular} & $3.4 e+41$ & $1.0374^{* * *}$ & 1.2865 & $1.0069^{*}$ \\
\hline & $(0.0051)$ & $(0.0010)$ & $(0.0008)$ & $(9.8 e+48)$ & $(0.0004)$ & $(2.4 e+04)$ & $(0.0001)$ \\
\hline $\begin{array}{l}\mathrm{PR}^{2} \\
\mathrm{p}\end{array}$ & \multicolumn{3}{|c|}{$\begin{array}{l}0.2524 \\
0.0000\end{array}$} & $\begin{array}{l}0.7421 \\
0.0000\end{array}$ & & $\begin{array}{l}0.3530 \\
0.0000\end{array}$ & \\
\hline
\end{tabular}

Nota: la categoría de referencia es dejar el sistema. Los errores estándares están entre paréntesis. * p $0.05 * *$ p $0.01 * * *$ p 0.001 (test de dos colas).

Del resultado de las estimaciones, vemos que para la primera transición de la cohorte de egresados en 1996 de EMCH el coeficiente de la variable rm en las tres categorías tiene el signo esperado, indicando que es más probable que se continúe estudios superiores (respecto a dejar el sistema) si se está ubicado en dicha región. Esto 
es consistente, considerando que la Región Metropolitana concentra ${ }^{11}$ gran parte de la oferta educativa. Aunque sólo es estadísticamente significativa para las categorías "ingresa a IP" y "ingresa a Ues".

La situación es distinta en la segunda transición, en la cual sólo es estadísticamente significativa en la categoría asistir a IP o Ues, lo que sigue siendo consistente con la explicación antes expuesta, y pareciera no tener tanta relación respecto del hecho de completar el ciclo iniciado anteriormente. Esto puede deberse a que la decisión de localización fue realizada en la transición anterior, y son otros factores los que determinan que el ciclo se culmine (académico, financiero, personal, entre otros). Algo similar ocurre en la tercera transición, aunque se puede notar que el efecto relativo de esta variable declina cuando nos movemos entre transiciones.

Respecto de la variable de educación del padre, en la primera transición las estimaciones arrojan resultados que parecieran ser contraintuitivos, indicando que para un cambio en una unidad de la variable epadre (un año de escolaridad) disminuirá el log ratio en un 15,41, 0,3 y 2,07 para las categorías "ingresar a CFT (aunque no es estadísticamente significativa), "ingresar a IP" y "ingresar a Ues", respectivamente. Efectos similares se mantienen en las transiciones posteriores. Si bien la evidencia sugiere que la escolaridad de los padres influye en buena parte del alcance educacional de los hijos, este resultado en cierta manera es robusto con los expuestos por Mare (1980), quien encuentra que los efectos de los grados de escolaridad de los padres van declinando desde las transiciones más tempranas de educación hacia aquellas que implican que el individuo acceda a educación superior.

La variable ingreso total per cápita en la primera transición posee el signo esperado y es estadísticamente significativa en las tres categorías, indicando que para un cambio en $\$ 1.000^{12}$ en la variable aumentará el log

11 Este hecho es aún más evidente si tenemos en cuenta que en el periodo bajo estudio todavía no se producía la diversificación de la oferta de educación superior que tuvo lugar en las regiones del país, aunque no deja de ser cierto que, no obstante esto, la mayor parte de la oferta sigue concentrada en la Región Metropolitana.

12 El nivel de ingreso está definido en miles de pesos chilenos. 
ratio en un 0,04,0,06 y 0,05 para la primera, segunda y tercera categoría respectivamente. El efecto merma en su importancia hacia el final de la trayectoria. Resultado que es consistente, ya que el acceso a educación superior está fuertemente correlacionado con el nivel socioeconómico de las familias y, en parte, con las restricciones al crédito.

La situación para los egresados de EMTP es un poco distinta:

Tabla 2: Ratio odds estimación del modelo: Cohorte egresados $4{ }^{\text {to }}$ Medio Técnico Profesional en 1996

\begin{tabular}{|c|c|c|c|c|c|c|c|}
\hline & \multicolumn{3}{|c|}{ T1 1996} & \multicolumn{2}{|c|}{ T2 2002} & \multicolumn{2}{|c|}{ T3 2006} \\
\hline & $\begin{array}{c}\text { Ingresa } \\
\text { a CFT }\end{array}$ & $\begin{array}{l}\text { Ingresa } \\
\text { a IP }\end{array}$ & $\begin{array}{l}\text { Ingresa } \\
\text { a Ues }\end{array}$ & $\begin{array}{c}\text { Completa } \\
\text { ciclo }\end{array}$ & $\begin{array}{l}\text { Continúa } \\
\text { Ues o IP }\end{array}$ & $\begin{array}{c}\text { Completa } \\
\text { ciclo }\end{array}$ & $\begin{array}{l}\text { Continúa } \\
\text { Ues o IP }\end{array}$ \\
\hline \multirow[b]{2}{*}{$\mathrm{rm}$} & 0.0000 & $2.0 e+61$ & $268.4847 * * *$ & 0.0000 & $4.6 e+32$ & $4.4 e+38$ & $60.4827^{* * *}$ \\
\hline & & & $(33.9758)$ & & & & (6.6609) \\
\hline \multirow{2}{*}{ epadre } & $367.3956^{* * *}$ & $0.0000^{* * *}$ & $0.3087^{* * *}$ & $0.0015^{* * *}$ & $0.0000^{* * *}$ & $0.0000^{\text {**** }}$ & $0.3581^{* * *}$ \\
\hline & (31.4181) & $(0.0000)$ & $(0.0102)$ & $(0.0003)$ & $(0.0000)$ & $(0.0000)$ & $(0.0276)$ \\
\hline \multirow{2}{*}{$\begin{array}{l}\text { Ingreso } \\
\text { total per } \\
\text { cápita }\end{array}$} & $0.7871^{* * *}$ & $0.9808^{* * *}$ & $0.9839 * * *$ & $1.4687^{* * *}$ & $1.2102 * * *$ & $1.0279 * * *$ & $1.0704 * * *$ \\
\hline & $(0.0258)$ & $(0.0008)$ & $(0.0005)$ & $(0.0213)$ & $(0.0054)$ & $(0.0007)$ & $(0.0012)$ \\
\hline $\begin{array}{l}\mathrm{PR}^{2} \\
\mathrm{p}\end{array}$ & & $\begin{array}{l}0.4331 \\
0.0000 \\
\end{array}$ & & $\begin{array}{l}0.8304 \\
0.0000\end{array}$ & & $\begin{array}{l}0.6347 \\
0.0000\end{array}$ & \\
\hline
\end{tabular}

Nota: la categoría de referencia es dejar el sistema. Los errores estándares están entre paréntesis. $*$ p $0.05 * *$ p $0.01 * * *$ p 0.001 (test de dos colas).

Para la primera transición la variable de localización tiene el signo esperado (con excepción de la primera categoría), pero no es estadísticamente significativa. Esto ocurre en las siguientes transiciones.

Al igual de lo que sucedía con los egresados de $\mathrm{EMCH}$ la escolaridad de los padres tiene el signo contrario al esperado a lo largo de las transiciones, aunque se distingue que en la primera transición tiene un importante impacto positivo sobre la primera categoría (ingresar a CFT).

La variable ingreso tiene el signo contrario al esperado en la primera transición para las tres categorías, esto es, a medida que aumenta el ingreso total per cápita, la probabilidad de acceder a la 
educación superior respecto de salir del sistema disminuye ${ }^{13}$. Una posible explicación es que estos egresados están trabajando al salir de la educación media, y que su ingreso representa una parte importante en el ingreso total de la familia. El ingreso de la ocupación principal de los que trabajaban al momento de realizar esta transición (40\%) representa alrededor de 50\% del ingreso autónomo del hogar.

La información para la cohorte 2001 se detalla a continuación:

Tabla 3: Ratio odds estimación del modelo. Cohorte egresados $4^{\text {to }}$ Medio Científico Humanista en 2001

\begin{tabular}{|l|c|c|c|c|c|}
\hline & \multicolumn{3}{|c|}{ T1 2002 } & \multicolumn{2}{c|}{ T2 2006 } \\
\hline \multirow{2}{*}{ rm } & Ingresa a CFT & Ingresa a IP & Ingresa a Ues & Completa ciclo & Continúa Ues o IP \\
\cline { 2 - 6 } & $1.3 e+09$ & 0.9894 & $0.1449 * * *$ & $0.6285^{* * *}$ & $0.1437^{* * *}$ \\
\hline \multirow{2}{*}{ epadre } & $0.2415^{* * *}$ & $(0.0443)$ & $(0.0047)$ & $(0.0208)$ & $(0.0043)$ \\
\cline { 2 - 6 } & $(0.0012)$ & $(0.0009)$ & $(3.5896)$ & $(0.0015)$ & $(9.2178)$ \\
\hline \multirow{2}{*}{$\begin{array}{l}\text { Ingreso total } \\
\text { per cápita }\end{array}$} & $1.0081^{* * *}$ & $1.0345^{* * *}$ & $1.0338^{* * *}$ & $1.0130^{* * *}$ & $1.0157^{* * *}$ \\
\cline { 2 - 6 } & $(0.0006)$ & $(0.0004)$ & $(0.0004)$ & $(0.0002)$ & $(0.0002)$ \\
\cline { 2 - 6 } PR $^{2}$ \\
p
\end{tabular}

Nota: la categoría de referencia es dejar el sistema. Los errores estándares están entre paréntesis. * p $0.05 * *$ p $0.01 * * *$ p 0.001 (test de dos colas).

En el caso de los egresados de EMCH, se observan diferencias en los patrones de influencia en las trayectorias respecto de la cohorte del 1996. La variable rm deja de tener un impacto significativo positivo, esto es, si alguien se encuentra en la Región Metropolitana tiene mayor probabilidad de dejar el sistema que acceder a las diferentes modalidades de educación superior, aunque el efecto encontrado no es estadísticamente significativo.

Hay también una distinción respecto de la influencia de la escolaridad de los padres en la trayectoria educativa de los hijos. Se mantienen los resultados de la cohorte anterior respecto de "ingresar a cft" e "ingresar a ip" en la primera transición, pero hay un efecto positivo importante en cuanto a "ingreso a la universidad", lo que

13 Lo que es lo mismo, aumenta la probabilidad de salir del sistema. 
muestra que, en este caso, el acceso a la universidad depende también de factores de ambiente.

Con relación a la influencia del ingreso per cápita, posee las mismas características analizadas en la cohorte 1996 y va disminuyendo sus efectos a medida que nos movemos en la trayectoria.

En el caso de los egresados de EMTP, al igual que en el caso de la cohorte 1996, la variable rm tiene un efecto ambiguo (véase en tabla 4).

Tabla 4: Ratio odds estimación del modelo. Cohorte egresados $4^{\text {to }}$ Medio Técnico Profesional en 2001

\begin{tabular}{|l|c|c|c|c|c|}
\hline & \multicolumn{3}{|c|}{ T1 2002 } & \multicolumn{2}{c|}{ T2 2006 } \\
\hline \multirow{2}{*}{ rm } & Ingresa a CFT & Ingresa a IP & Ingresa a Ues & Completa ciclo & Continúa Ues o IP \\
\cline { 2 - 6 } & 0.0000 & $1.4533^{* * *}$ & $0.2523^{* * *}$ & $10.8020^{* * *}$ & $0.7454^{* * *}$ \\
\cline { 2 - 6 } & $(0.0000)$ & $(0.0533)$ & $(0.0077)$ & $(0.7924)$ & $(0.0229)$ \\
\hline \multirow{2}{*}{ epadre } & $2.7647^{* * *}$ & $1.0 e+03^{* * *}$ & $508.7150^{* * *}$ & $1.5 e+03^{* * *}$ & $1.4 e+03^{* * *}$ \\
\cline { 2 - 6 } & $(0.0325)$ & $(3.7186)$ & $(1.4277)$ & $(10.8044)$ & $(4.135)$ \\
\hline Ingreso total & $0.9561^{* * *}$ & $1.0215^{* * *}$ & $1.0211^{* * *}$ & $1.0060^{* * *}$ & $1.0017^{* * *}$ \\
\cline { 2 - 6 } per cápita & $(0.0038)$ & $(0.0003)$ & $(0.0003)$ & $(0.0003)$ & $(0.0003)$ \\
\cline { 2 - 6 } PR & & 0.1263 & & 0.0548 & \\
p & & 0.0000 & & 0.0000 & \\
\hline
\end{tabular}

Nota: la categoría de referencia es dejar el sistema. Los errores estándares están entre paréntesis. * p $0.05 * *$ p $0.01 * * *$ p 0.001 (test de dos colas).

Respecto de la variable escolaridad de los padres, hay una influencia positiva que afecta a las probabilidades de acceder a educación superior en relación con las de dejar el sistema. Esta influencia es mayor cuando se analiza el caso de acceder a IP o Ues. Se mantiene este efecto en la siguiente transición.

La variable ingreso posee las características que hemos mencionado antes.

\section{Trayectorias laborales}

Respecto de la situación laboral, se compararon las cohortes bajo estudio en dos años en el tiempo: 2001 y 2006. A continuación, se analiza a los egresados de EMCH para la cohorte 1996. 
En términos generales de tales egresados, en 2001 un 19\% estaba trabajando versus un $81 \%$ que no lo hacía; $97 \%$ de éstos declara no estar buscando trabajo y un $95 \%$ no lo hace por estar estudiando. Un 60\% es mujer, y el 93\% está soltero. Un 97\% declara no haber asistido a cursos de capacitación. Un 52,7\% trabaja como técnico especialista, un $36,3 \%$ es empleado de comercio, vendedor, un $8 \%$ operario en el sector industrial y el $3 \%$ restante trabajador por cuenta propia. El 57,3\% posee trabajo permanente.

$\mathrm{Al}$ año 2006, 60\% estaba trabajando versus un $40 \%$ que no lo hacía; $75,6 \%$ de éstos declara no estar buscando trabajo y $61,6 \%$ no lo hace por estar estudiando. Un 37,2\% declara haber asistido a cursos de capacitación a través de la empresa en que trabaja. Un 86,3\% es profesional universitario, $9 \%$ empleado de comercio, vendedor y el $4,7 \%$ restante operario en el sector industrial.

Teniendo en cuenta la trayectorias educativas, los que ingresaron en la primera (1997) en $\mathrm{CFT}^{14}$ 100\% no trabajaba; al 2006 un 38,7\% de éstos estaba trabajando como vendedor en el comercio.

De los que ingresaron a IP ${ }^{15}, 77 \%$ trabajaba en 2001 y lo hacía como técnico/especialista. Para $2006^{16}$ sólo lo hacía un 22,1\%, un $51 \%$ en el campo profesional y el restante como vendedor.

De los que ingresaron a la universidad ${ }^{17}$ ese año, a 2001 no estaban trabajando y a 2006, 68,7\% declaran desempeñarse como profesionales en diferentes áreas.

Respecto de los que dejaron el sistema para ese año ${ }^{18}, 71,2 \%$ trabajaba en 2001 y para 2006 esta cifra fue de 48,7\%, trabajando en las mismas áreas, técnicos especialistas, empleados de comercio y vendedores y operarios industriales.

\footnotetext{
14 En la siguiente trayectoria 2002 estaban fuera del sistema.

15 Completaron ciclo en la trayectoria posterior.

16 Los que continuaron en el sistema a 2002.

17 Continuaron en la universidad en la trayectoria posterior.

18 Continuaron fuera del sistema educativo en la siguiente trayectoria.
} 
En relación con el ingreso percibido en ambos años, del 4,5\% que estaban trabajando ambos años (2001 y 2006), 67,4\% aumentó su nivel de ingreso recibido por la ocupación principal. Hay que destacar que todos los que estaban trabajando en ambos años salieron del sistema en la primera transición, y los que disminuyeron su nivel de ingreso trabajaban como peones.

Respecto de la cohorte 1996 de egresados de EMTP, en 2001 un $82,3 \%$ estaba trabajando versus un $17,7 \%$ que no lo hacía; $71,8 \%$ de éstos declara no estar buscando trabajo y un $71 \%$ no lo hace por estar estudiando. Un 64\% es mujer, y el 93\% está soltero. Un 99,5\% declara no haber asistido a cursos de capacitación. Un 29,5\% trabaja como empleado de contabilidad, un 28,5\% como operario industrial, un $15,4 \%$ en oficios, un $13,1 \%$ como técnico, maestro y especialista, un $6,9 \%$ como conductores y el restante $6,6 \%$ como vendedor de tienda. El 68,8\% posee trabajo permanente.

$\mathrm{Al}$ año 2006, un 91,4\% estaba trabajando versus un 8,6\% que no lo hacía; $100 \%$ de éstos declara no estar buscando trabajo; un $91,9 \%$ no lo hace por dedicarse a los quehaceres del hogar y el resto está estudiando. Un 21,2\% declara haber asistido a cursos de capacitación a través de la empresa en que trabaja. Un 20,4\% es profesional universitario, un 44,6\% empleado de comercio, vendedor, el $11,4 \%$ se dedica a la construcción y el porcentaje restante se reparte entre operarios en el sector industrial, técnicos especialistas y conductores.

Teniendo en cuenta las trayectorias educativas, los que ingresaron en la primera (1997) en $\mathrm{CFT}^{19}$, el 100\% trabajaba como técnico especialista; al 2006 continúan trabajando como mecánicos.

De los que ingresaron a $\mathrm{IP}^{20}, 100 \%$ trabajaba en el 2001, un $50,4 \%$ de éstos lo hacía como técnico y especialista y el resto desarrollaba un oficio; para 2006 continuaban trabajando la totalidad en las mismas áreas.

19 En la siguiente trayectoria 2002 completó el ciclo.

20 El 49,6\% completaron ciclo en la trayectoria posterior (2002) y un 50,1\% continuó estudiando. 
De los que ingresaron a la universidad ${ }^{21}$ ese año, al 2001 un $42 \%$ estaba trabajando como empleado de contabilidad, y a 2006 un 95,8\% declara hacerlo y lo hace como profesional.

Respecto de los que dejaron el sistema ${ }^{22}$, para ese año un $89,1 \%$ trabajaba en el 2001 como técnico, operario, en oficios y como vendedor; para 2006 se mantienen en los mismos tipos de trabajos.

En relación con el ingreso percibido en ambos años, del $65,1 \%$ que estaba trabajando ambos años (2001 y 2006), el 90,7\% aumentó su nivel de ingreso recibido por la ocupación principal. Al igual que en el caso de EMCH, los que disminuyeron su nivel de ingreso dejaron el sistema de educación en la primera transición y trabajaban como peones y vendedores.

La situación laboral para la cohorte 2001 de egresados de EMCH se detalla a continuación. Si bien la trayectoria en esta cohorte es más corta en cuanto a tiempo, por lo que analizar dinámicas o cambios de estados no será posible, resulta interesante reportar algunos resultados.

En el año 2001, el 100\% no trabajaba; declara no estar buscando trabajo y no lo hace por estar estudiando. Un 60\% es mujer y en su totalidad soltero. Un 98,6\% declara no haber asistido a cursos de capacitación.

Al 2006, un 36,1\% estaba trabajando versus un 63,9\% que no lo hacía; $75,8 \%$ de éstos declara no estar buscando trabajo, un 66,3\% no lo hace porque declara estar estudiando, un $28,5 \%$ por dedicarse a los quehaceres del hogar y el resto porque no le interesa. Un 91\% declara no haber asistido a cursos de capacitación. Un 30,6\% trabajaba en algún oficio, un $21,7 \%$ es empleado de comercio, vendedor, el $14,1 \%$ se dedica a la agricultura, un $14 \%$ técnico especialista, un $6,7 \%$ profesional y el porcentaje restante se reparte entre operarios en el sector industrial y conductores.

21 Continuaban en la universidad en la trayectoria posterior.

22 Continuaron fuera del sistema educativo en la siguiente trayectoria. 
Teniendo en cuenta la trayectorias educativas, los que ingresaron en la primera (2002) en $\mathrm{CFT}^{23}$, el 77,8\% trabajaba como técnico especialista.

De los que ingresaron a $\mathrm{IP}^{24}$, un 71,8\% trabajaba en el 2006, un $7 \%$ de éstos desarrollaba un oficio y el resto está ocupado en el sector público.

De los que ingresaron a la universidad ${ }^{25}$ ese año, al 2006 un $11 \%$ estaba trabajando como profesional.

Respecto de los que dejaron el sistema ${ }^{26}$ para ese año, un 42,6\% trabajaba en el 2006 como técnico, operario, vendedor y en la agricultura.

\section{Conclusiones y comentarios}

A raíz de la discusión en torno a la situación de la educación técnica en Chile, se propuso un análisis de las trayectorias educativas de los individuos, con la inquietud, en primer lugar, de observar si este tipo de educación fue una opción al momento de escoger una alternativa de educación superior. No se buscó analizar los determinantes de proseguir con estudios superiores, ya que es sujeto de otra investigación y va más allá del alcance de este estudio. Se pretendió analizar cómo los miembros de las diferentes cohortes elegidas transcurrieron a través de su trayectoria educativa, además de observar si patrones del origen social de los individuos influyeron en la senda educativa que escogió. Se encontró que las variables de localización en la Región Metropolitana y el ingreso total per cápita fueron determinantes en explicar las transiciones que siguieron los individuos en su proceso educativo.

23 En la siguiente trayectoria 2006 completó el ciclo.

24 El 15,4\% completó ciclo en la trayectoria posterior (2006), un 30,8\% continuó estudiando y un $53,9 \%$ dejó el sistema.

25 Un $73,6 \%$ continuaba en la universidad en la trayectoria posterior, un 25\% completó el ciclo y un 1,4\% dejó el sistema.

26 Continuaron fuera del sistema educativo en la siguiente trayectoria. 
Respecto a la situación laboral, a modo de conclusión se puede determinar que, en general, los egresados de la EMCH comienzan a trabajar en forma más tardía debido principalmente a que permanecen en el sistema educativo, principalmente en la universidad. Los primeros trabajos son en su gran mayoría temporales y después se insertan en el mercado realizando empleos profesionales.

Con relación a las personas que salieron del sistema antes de la primera transición, existe una tendencia a permanecer en las mismas tareas (se divisa en la cohorte 1996), en empleos de menor calificación. Incluso, cuando se analiza el cambio en el ingreso en los que trabajaban en ambos años, son estas personas las que presentan, en algunos casos, empeoramientos en el ingreso percibido.

En cambio, los egresados de EMTP muestran un porcentaje mayor de participación en el mercado laboral en etapas más tempranas de la trayectoria. Trabajan mientras estudian y sus ocupaciones son más variadas que las de los EMCH. Al igual que en el caso anterior, los que empeoran en su nivel de ingreso percibido son aquellos que no continuaron estudiando y cuyos empleos son de baja calificación.

La trayectoria educativa y laboral es un objeto de investigación complejo por ser un fenómeno multivariado, que se desarrolla como proceso y con componentes de subjetividad del actor. Enfocarnos en el análisis de trayectorias nos da idea de la evolución y es, por tanto, un insumo más útil a la hora de tomar decisiones de intervención vía políticas públicas. En el caso de la educación técnica hay mucho terreno que recorrer, principalmente porque hay poco estudiado y reportado en la materia, y mucho menos medido, lo que constituye una gran dificultad a la hora de intentar profundizar en esta problemática. Sería bueno medir está dinámica para distintos años en que se aplicaron medidas de políticas, por ejemplo, el caso de la Beca Nuevo Milenio y del crédito Ley 20.027, para ver si se está potenciando la educación técnica verdaderamente y si se llega con oportunidades reales a aquellos sectores que pretende abarcar.

Recibido: 29 de septiembre de 2008 Aceptado: 27 de octubre de 2008 